\title{
GEO
}

Geograficando

ISSN: 2346-898X

geograficando@fahce.unlp.edu.ar

Universidad Nacional de La Plata

Argentina

\section{Metodología para el diagnóstico de cuencas hídricas. El caso de la región La Plata, Argentina}

Jensen, Karina; Cremaschi, María Elisa; Freaza, Nadia

Metodología para el diagnóstico de cuencas hídricas. El caso de la región La Plata, Argentina

Geograficando, vol. 16, núm. 1, 2020

Universidad Nacional de La Plata, Argentina

DOI: https://doi.org/10.24215/2346898Xe069

Atribución no comercial compartir igual (CC BY-NC-SA) 4.0 
Artículos

\section{Metodología para el diagnóstico de cuencas hídricas. El caso de la región La Plata, Argentina}

Methodology for hydrological basin diagnostics. The case of the region of La Plata, Argentina

Karina Jensen

DOI: https://doi.org/10.24215/2346898Xe069

Instituto de Politicas del Ambiente Construido IIPAC

(FAU UNLP), Argentina

karinacjensen@gmail.com

Recepción: 05 Agosto 2019

Maria Elisa Cremaschi

Aprobación: 15 Abril 2020

Instituto de Politicas del Ambiente Construido IIPAC

(FAU UNLP), Argentina

elisacre@hotmail.com

Nadia Freaza

Instituto de Políticas del Ambiente Construido IIPAC

(FAU UNLP), Argentina

freazanadia@gmail.com

Recepción: 05 Agosto 2019

Aprobación: 15 Abril 2020

\section{Resumen:}

Este trabajo tiene por finalidad desarrollar una metodología de diagnóstico para abordar desde una visión holística la problemática de las inundaciones. Se toman como caso de estudio las cuencas hídricas en la Región del Gran La Plata. La metodología se compone de dos momentos: un primer momento de relevamiento y análisis de todas las cuencas de la región, y un segundo momento de diagnóstico para identificar conflictos y potencialidades. A partir de reconocer la complejidad del territorio y la multidimensionalidad del problema, se construye una matriz de análisis para determinar el grado de vulnerabilidad del sistema de cuencas hídricas. Se construye así una metodología posible de ser replicable en otras regiones con riesgo de inundaciones, con el fin de prevenir y mitigar su impacto.

Palabras Clave: Metodología, Inundación, Construcción social del riesgo, Vulnerabilidad.

\section{Abstract:}

The purpose of this paper is to develop a diagnostic methodology to approach the problem of floods from a holistic perspective. Hydrological basins in the Gran La Plata Region are taken as a case study. The methodology is composed of two stages: a first stage of survey and analysis of all the basins of the region, and a second diagnostic stage to identify conflicts and potentialities. After recognizing the territory's complexity and the problem's multidimensionality, an analysis matrix is constructed to determine the watershed system's degree of vulnerability. Thus, this methodology can be replicated in other regions with flooding risk in order to prevent and mitigate flooding impact.

KEYWORDS: Methodology, Flooding, Social construction of risk, Vulnerability.

\section{INTRODUCCIÓN}

Uno de los fenómenos que demuestran los desequilibrios territoriales y las problemáticas ambientales de la Región Gran La Plata (RGLP) son las inundaciones, ya sea por las sucesivas crecidas del Río de la Plata (formado por los ríos Paraná y Uruguay, de $290 \mathrm{~km}$ de largo y una anchura máxima es de $219 \mathrm{~km}$ ) o lluvias extraordinarias en un corto lapso de tiempo. En los últimos 20 años la RGLP se vio afectada por sucesivas inundaciones. En el año 2002 cayeron casi 80 milímetros en una hora; en marzo de 2005 la región se vio 
nuevamente afectada, situación que se repitió en febrero de 2008, cuando por las grandes precipitaciones 90.000 personas fueron afectadas. Se puede decir sin duda que el punto más crítico alcanzado en la región fue la catástrofe del 2 de abril del 2013, cuando llovieron casi $400 \mathrm{~mm}$ en menos de 6 horas, lo que afectó a gran parte de la población y atravesó todos los estratos sociales.

Los efectos anteriormente descritos son el disparador para desarrollar un diagnóstico integral con el fin de prevenir y mitigar el impacto de inundaciones sobre contextos urbanos y periurbanos en toda su complejidad, en pos de establecer una nueva relación agua - ciudad. Todo ello, a partir de incrementar la resiliencia urbana con mecanismos que mejoren las condiciones del ambiente y de la infraestructura de la cuenca, la articulación interjurisdiccional y la incorporación de mecanismos que incentiven la participación ciudadana para contribuir a la concientización ambiental de la población en general.

El marco teórico conceptual que se utilizó para abordar el presente trabajo recoge diversos conceptos teóricos con el fin de pensar las problemáticas de las cuencas de la RGLP desde un enfoque integral. Dichos conceptos permiten instrumentar herramientas teórico-prácticas, tanto para el análisis del diagnóstico como para el desarrollo posterior de estrategias de intervención.

\section{EL RIESGO COMO CONSTRUCCIÓN SOCIAL}

Los llamados desastres naturales son fenómenos propios de la naturaleza, de mayor o menor intensidad, pero inevitables e incontrolables por el ser humano. Sin embargo, la exposición a dichos fenómenos es un factor que el hombre puede controlar. Como señalan Hilda Herzer y Di Virgilio (1996),

hace unas décadas comenzó a desarrollarse una nueva perspectiva en las ciencias sociales para analizar los desastres, en contraposición al enfoque de las ciencias naturales y fisicas, que plantea interpretar a los desastres no ya como elementos geofisicos aislados, singulares y extremos, sino como manifestaciones de un proceso social continuo que impactan en las condiciones cotidianas de la vida de una sociedad.

De esta manera, se puede entender que el riesgo de sufrir un desastre ya no depende solamente de la magnitud del fenómeno natural sino que se relaciona con la vulnerabilidad de la sociedad expuesta a la amenaza.

Esta corriente de pensamiento parte de la idea de que los fenómenos de la naturaleza no son un problema per se, sino que se constituyen en un problema a partir de la relación entre lo natural y la organización y estructura de la sociedad, y de cómo se establece dicha relación. En consecuencia, se parte del principio de que los desastres no son un problema independiente coyuntural o excepcional sino que tanto los procesos causales como las estrategias reales y viables para superarlos atañen a la sociedad y a los procesos de desarrollo en sí. En este sentido, se quiere enfatizar la naturaleza social del problema, remarcando que la construcción del riesgo es un proceso social e histórico. "El desastre es un proceso y un producto que manifiesta el nivel de riesgo que existe en una sociedad. Es la expresión más evidente de una convivencia vulnerable entre diversos grupos sociales y su medio" (Herzer, 2011).

Si bien el impacto de las diversas amenazas físicas con que convive el hombre en este planeta y de los desastres que suscitan es creciente, en términos del impacto material como también de vidas humanas, para pensar en la prevención o la reducción los daños la atención focalizada en los fenómenos de gran impacto resulta uno de los errores conceptuales y metodológicos más generalizados. La mirada orientada desde la concepción de la construcción social del riesgo corre el eje de análisis del fenómeno natural y plantea el estudio a partir de tres conceptos: riesgo, amenaza y vulnerabilidad. Estos conceptos son fundamentales a la hora de comprender el origen de los problemas y plantean no soluciones definitivas, sino el manejo de los desastres.

Tomando las definiciones de UNISDR ${ }^{1}$, se entiende por riesgo la combinación de la probabilidad de que se produzca un hecho y sus consecuencias negativas. Los factores que lo componen son la amenaza y la vulnerabilidad. 
Por un lado, amenaza es un fenómeno, sustancia, actividad humana o condición peligrosa que puede ocasionar la muerte, lesiones u otros impactos a la salud, al igual que daños a la propiedad, la pérdida de medios de sustento y de servicios, trastornos sociales y económicos, o daños ambientales. La amenaza se determina en función de la intensidad y la frecuencia. Y por otro, por vulnerabilidad entendemos las características y las circunstancias de una comunidad, sistema o bien que los hacen susceptibles a los efectos dañinos de una amenaza. Con los factores mencionados se compone la siguiente fórmula de riesgo: amenaza x vulnerabilidad. La vulnerabilidad de las comunidades es el factor más crítico a tener en cuenta, ya que en algunos casos la exposición a las amenazas y la baja capacidad de resiliencia de las comunidades se constituyen como factores de riesgo incluso frente a fenómenos de baja intensidad (Figura 1). Una visión amplia sobre el medio ambiente urbano debería partir del reconocimiento de la fragmentación social de la ciudad como factor de vulnerabilidad social y ambiental. La vulnerabilidad puede ser definida como la propensión o susceptibilidad de la sociedad, los seres humanos y sus soportes productivos, infraestructurales o materiales a sufrir daños y pérdidas cuando son impactados por hechos o fenómenos físicos externos, y de encontrar dificultades en recuperarse posteriormente, de manera autónoma (Lavell, 2004). Es decir, tanto la exposición a una amenaza como la capacidad de resiliencia urbana son elementos a considerar en el análisis en términos de afectación de desastres.

FIGURA 1

Cuadro de conceptos y fórmulas

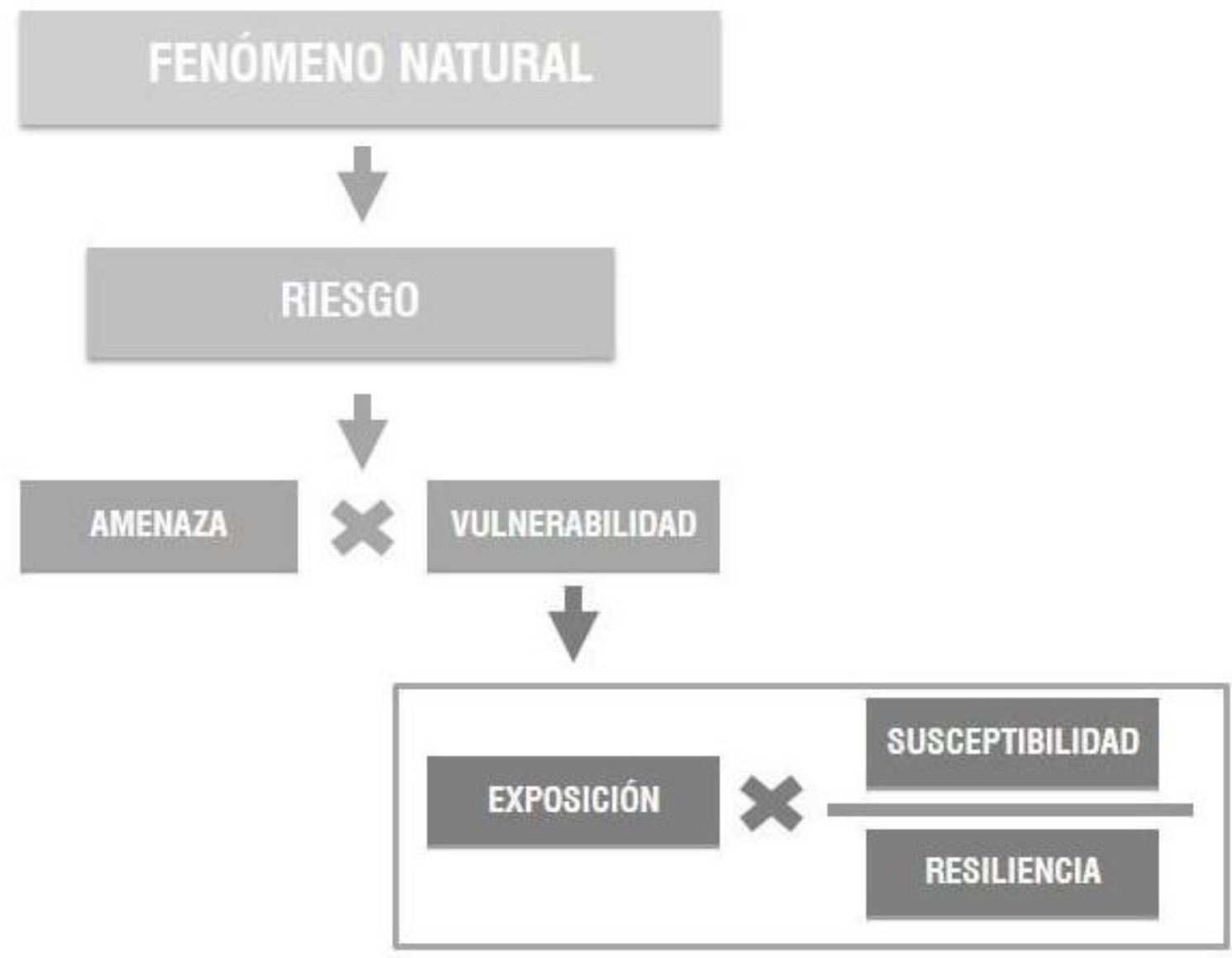

Elaboración propia, 2019

Estos conceptos permiten orientar el análisis para la identificación de tres puntos fundamentales: i) las relaciones entre la sociedad y su medio, en términos de vulnerabilidad, a los efectos de amenazas presentes en el medio; ii) las relaciones entre diversos actores sociales; y iii) las articulaciones entre distintos niveles 
jurisdiccionales y sectoriales que se establecen en el territorio, en términos de capacidad de resiliencia urbana frente al riesgo. Dichas relaciones permitirán establecer la escala de análisis a aplicar en el estudio de la construcción del riesgo hídrico. En este sentido, se hace evidente que no alcanza con utilizar únicamente el territorio como unidad de análisis, sino que se hace necesario añadir otras categorías que incorporen las características de la sociedad. Para comprender cómo es la sociedad es indispensable ver dónde se asientan quienes la integran, cómo se compone esa población, el nivel socioeconómico y la densidad.

De esta manera, se comprende la inundación de abril de 2013 como la manifestación de los desequilibrios entre la sociedad, que tiene una cultura y un proceso histórico particular en la ciudad de La Plata, y el medio, que tiene características geofísicas y medioambientales propias de la llanura costera de la pampa ondulada.

\section{Metodología}

La metodología implementada se compone de dos momentos: un primer momento de relevamiento y análisis de todas las cuencas de la región del gran La Plata, que permitió conocer la región, y un segundo momento de diagnóstico de las cuencas desde el concepto de construcción social del riesgo, que permitió identificar los principales conflictos y problemáticas frente a la amenaza de inundación, para determinar la vulnerabilidad de la población.

El primer momento de conocimiento de la RGLP se estructuró en etapas de relevamiento y análisis, tomando como objeto de estudio las cuencas hídricas que componen la región (Figura 2). Para la instrumentación de la metodología de análisis se desarrollaron diversas actividades: reconocimiento del área de estudio y visita de campo, recopilación de información, identificación y clasificación de fuentes, procesamiento de datos en SIG, sistematización de datos cuali-cuantitativos.

A partir de la concepción del territorio como sistema complejo, se tomó como unidad de análisis el área de las cuencas hídricas y se realizó un abordaje integral en el que se interrelacionan dimensiones naturales, urbanas, sociales, económicas, principalmente. Para medir estas dimensiones se componen de indicadores, de la dimensión natural son la orientación del arroyo, tipo de cauce, longitud y área. La dimensión urbana se evalúa con el porcentaje de urbanización de la cuenca; la dimensión social, con la composición de la población y con qué tipo de problemáticas socioambientales se observan en la cuenca; y la económica, con el nivel socioeconómico de la población.

El segundo momento está compuesto por una síntesis del análisis por cuenca que permitió identificar los principales conflictos para la construcción de un árbol de problemas. Para abordar el análisis se trabajó en el marco teórico con los conceptos de construcción social del riesgo, a partir del cual el problema se distancia del análisis del fenómeno natural para focalizarse en la vulnerabilidad socio-territorial. Este enfoque permitió desarrollar una matriz de análisis para determinar el grado de vulnerabilidad de las cuencas frente a inundaciones.

Los factores causales para determinar la vulnerabilidad se componen de la exposición, la susceptibilidad y la resiliencia. La exposición está determinada por la ocupación de la planicie de inundación, el porcentaje de impermeabilización de superficies, cuán alterado está el cauce natural, si hay contaminación hídrica y barreras que alteren el libre escurrimiento, y por último si en la cuenca hay basurales sobre la planicie de inundación. Todos ellos son factores que impiden el libre escurrimiento de las aguas. La susceptibilidad está relacionada con las características de la población de la cuenca que incrementan la exposición o disminuyen la capacidad de respuesta, como el nivel socio económico de la población, la densidad de población, el porcentaje de población en grupos de riesgo, el nivel educativo, la cantidad de población con NBI y la calidad constructiva de las viviendas. Por último, la resiliencia está dada por el nivel de preparación de la infraestructura y la gestión urbana para enfrentar el riesgo; en términos de infraestructura, consiste en la existencia de infraestructura y equipamiento para la contingencia, si existen obras hidráulicas para disminuir los efectos de las inundaciones, si la cuenca posee superficies urbanas permeables, si la cuenca posee parques de laminación, reservorios y 
retardadores que absorban el excedente de agua, si se les realiza mantenimiento a los sistemas de drenaje, si hay un sistema de monitoreo y vigilancia hidrometeorológico. En cuanto a la gestión urbana, está en relación con el acceso a la información que tienen los habitantes ante una inundación, la participación ciudadana, si hay un comité operativo de emergencias y, lo más importante, si ante una inundación hay un plan de contingencia, como también si se asesora y capacita a la población para que esté preparada para las inundaciones.

\section{LA REGIÓN DE ESTUDIO Y SU SISTEMA DE ARROYOS}

Los arroyos de la vertiente del Río de la Plata tienen un rumbo general de escurrimiento SO-NE, que antiguamente desembocaba en la planicie costera y no en el Río de la Plata. Ello se debía a que entre los 5 metros sobre el nivel del mar y la costa del Río de la Plata se produce un cambio de pendiente regional, la cual se reduce a $<0,03 \%$; ello da lugar a que los arroyos pierdan energía y sus cursos se hagan divagantes, lo que genera un área de bañados. Esta situación se revierte debido a la instalación de asentamientos urbanos, que implicó la elevación del nivel de terreno del bañado y la canalización de muchos de los cursos de agua para agilizar el escurrimiento y el desagüe al Río de la Plata. 
FIGURA 2

Cuencas de la RGLP

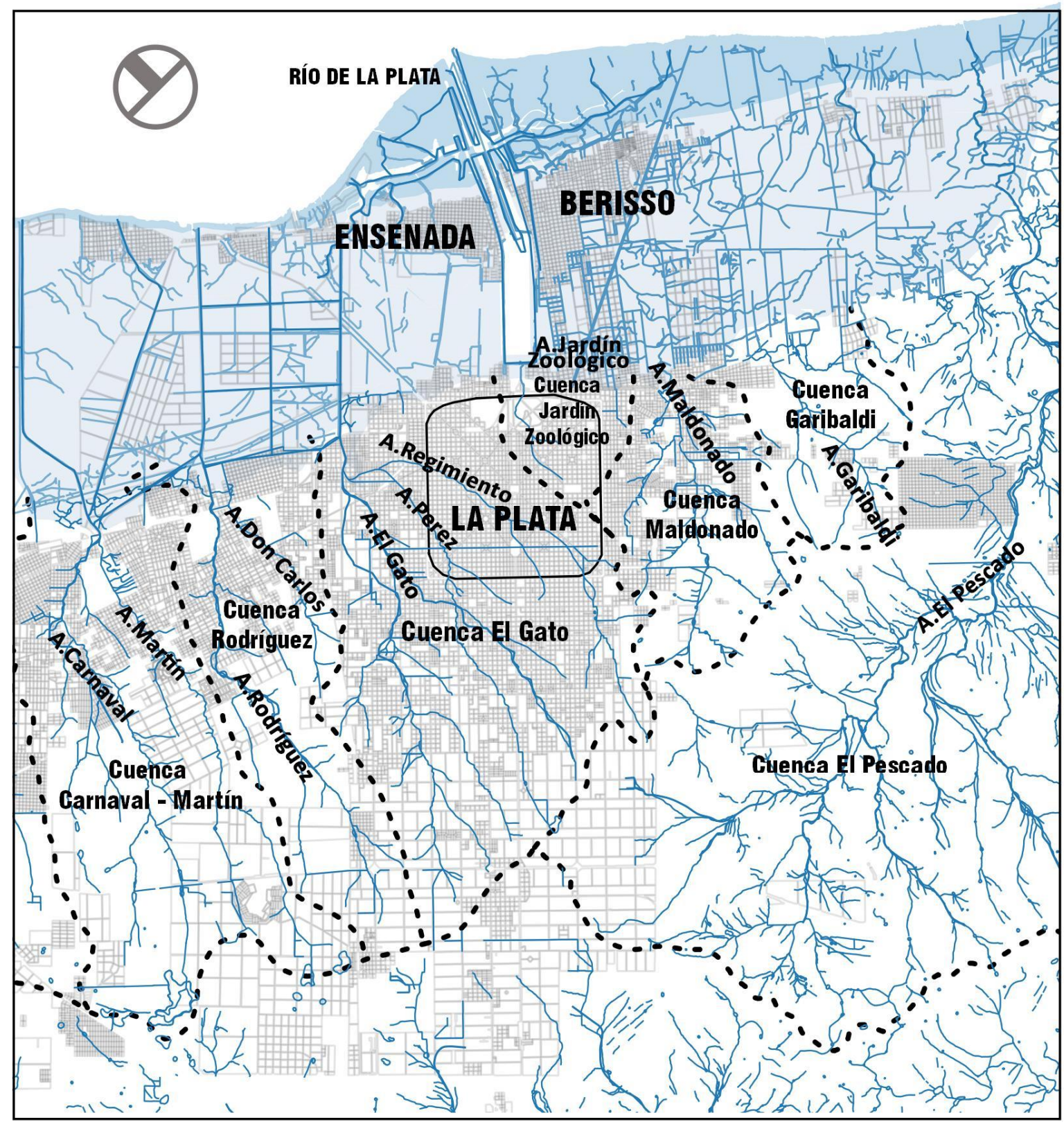

Elaboración propia, 2019

Todos los arroyos y cuerpos de agua presentan una baja calidad de sus aguas debido a la actividad humana, ya que se encuentran afectados por los efluentes de las diversas actividades industriales, principalmente por vertidos industriales (cobre, mercurio y plomo, hidrocarburos alifáticos y aromáticos, fenoles; grasas y aceites: benceno, naftalenos, antracenos y toluenos). Esto residuos son descargados en forma directa en los cursos de agua, sobre todo en los canales Este y Oeste que circundan el polo petroquímico, que son uno de los emblemas de la contaminación regional. Otro de los problemas que se encuentran en los arroyos y canales es la ocupación de las márgenes con asentamientos irregulares. Estos, aparte de ser propensos a la inundación, generan residuos que contaminan los canales.

Se pueden identificar tres grupos de arroyos: 
- Arroyos del norte: Carnaval, Martín, Rodríguez, Don Carlos y El Gato. Actúan como barreras o fracturas del tejido urbano.

- Arroyos dentro del tejido urbano fundacional: Pérez, Regimiento y Jardín Zoológico. Junto con sus tributarios, tienen gran influencia en la morfología de la ciudad. Los arroyos localizados al $S$ y SE tienen importante influencia sobre la morfología de la zona periurbana y, al ingresar al tejido fundacional, se encuentran entubados, por lo que pierden visibilidad y reconocimiento.

- Arroyos del sur: Circunvalación, Maldonado, Garibaldi y El Pescado. La periferia sur cuenta con menor grado de consolidación, por lo que los cursos tienen bajos grados de intervención. Sin embargo, actúan, al igual que en la zona norte, como barreras al crecimiento urbano.

A su vez, los arroyos se dividen en subcuencas: alta, media y baja. Cada una presenta características particulares en relación con el contexto que atraviesan, como grado de intervención del cauce, tipo de vegetación, usos, estado y calidad del curso superficial, interferencias, contaminantes, estado sanitario, entre otros. En términos generales, en la cuenca alta predominan la zona rural y el uso flori-hortícola intensivo. En la cuenca media se presenta mayor densidad poblacional, industrial y comercial, que en la mayoría de los casos se ve acompañada por un elevado grado de contaminación y de asentamientos informales sobre la planicie de inundación. Por último, la cuenca baja consiste, por lo general, en tramos agregados para mejorar el escurrimiento, que atraviesan la zona de bañados no urbanizada. 
TABLA 1

Características del sistema de cuencas

\begin{tabular}{|c|c|c|}
\hline & CUENGAS & CARÁGTERISTICAS \\
\hline 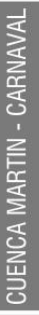 & $\begin{array}{l}Q \\
2 \\
(2)\end{array}$ & $\begin{array}{l}\text { Orientación } \\
\text { SO-NE } \\
\text { Área (ha) } \\
8.140 \\
\text { Cauce } \\
\text { Carnaval: Segundo orden Martin:Primer orden } \\
\text { Longitud (km) } \\
\text { Carnaval: } 18,5 \text { Martin:14,5 } \\
\text { Porcentaje urbanización: } \\
\text { Carnaval:15-20\% Martin: }\end{array}$ \\
\hline 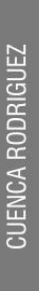 & 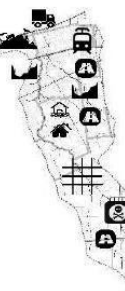 & $\begin{array}{l}\text { Orientación } \\
\text { SO-NE } \\
\text { Área (ha) } \\
5.430 \\
\text { Cauce } \\
\text { Rodriguez: Segundo orden Don Carlos:Primer orden } \\
\text { Longitud (km) } \\
\text { Rodriguez: } 15,2 \text { Don Carlos:4 } \\
\text { Porcentaje urbanización: } \\
30 \%\end{array}$ \\
\hline 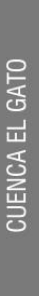 & 60 & $\begin{array}{l}\text { Orientación } \\
\text { SO-NE } \\
\text { Área (ha) } \\
12.412 \\
\text { Cauce } \\
\text { El Gato: Tercer orden Perez: Segundo orden Regimiento: Primer orden } \\
\text { Longitud (km) } \\
\text { El Gato: } 25 \text { Perez: } 12 \text { Regimiento: } 10 \\
\text { Porcentaje urbanización: } \\
\text { El Gato: } 70 \% \text { Perez: } 70 \% \text { Regimiento: } 80 \%\end{array}$ \\
\hline 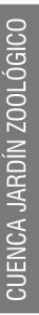 & & $\begin{array}{l}\text { Orientación } \\
\text { SO-NE } \\
\text { Área (ha) } \\
999 \\
\text { Cauce } \\
\text { Principal } \\
\text { Longitud (km) } \\
4 \\
\text { Porcentaje urbanización: } \\
95 \%\end{array}$ \\
\hline 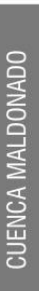 & & $\begin{array}{l}\text { Orientación } \\
\text { SO-NE } \\
\text { Área (ha) } \\
3.560 \\
\text { Cauce } \\
\text { Principal } \\
\text { Longitud (km) } \\
8 \\
\text { Porcentaje urbanización: } \\
40 \%\end{array}$ \\
\hline 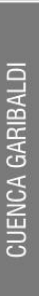 & & $\begin{array}{l}\text { Orientación } \\
\text { SO-NE } \\
\text { Área (ha) } \\
2.842 \\
\text { Cauce } \\
\text { Principal } \\
\text { Longitud (km) } \\
7,5 \\
\text { Porcentaje urbanización: } \\
70 \%\end{array}$ \\
\hline 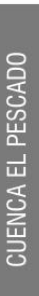 & 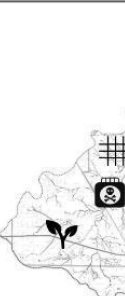 & $\begin{array}{l}\text { Orientación } \\
\text { SO-NE } \\
\text { Área (ha) } \\
34.900 \\
\text { Cauce } \\
\text { Principal } \\
\text { Longitud (km) } \\
40 \\
\text { Porcentaje urbanización: } \\
5 \%\end{array}$ \\
\hline
\end{tabular}




\section{LOS ARROYOS URBANOS Y SUS CONFLICTOS}

El diagnóstico se encuentra enfocado en la dimensión urbano-ambiental, ya que en el relevamiento se pudo observar que dicha dimensión es muy importante, dadas las condiciones geomorfológicas de la región de estudio, los cursos de agua y el desarrollo urbano. Se identificaron varios conflictos, como el : proceso de asentamiento de viviendas carenciadas que ocupan la ribera y su planicie de inundación del curso, lo que genera un elevado riesgo de inundación, que conlleva una importante diversidad de conflictos ambientales. Además, algunas de las estructuras de una margen a otra de ciertos arroyos ponen en riesgo de accidente a las personas, principalmente a niños pequeños y ancianos.

Se identificaron vuelcos directos en cuencas medias, extendidas o específicas, de residuos sólidos urbanos (RSU). La presencia de residuos, la vegetación abigarrada y el posterior estancamiento de agua promueven el desarrollo de plagas y vectores de enfermedades transmisibles al hombre. Mientras que el vuelco directo de aguas servidas desde los domicilios particulares y los vuelcos de efluentes cloacales directos o indirectos, con tratamiento deficiente o sin él, aportan microorganismos que pueden ser causantes de múltiples patologías humanas, desde parasitosis a infecciones bacterianas.

A esta situación se suma que varios pobladores no perciben el riesgo sanitario y ambiental al que se encuentran expuestos. Hay un uso del agua por parte de los niños para actividades recreativas y no existen suficiente educación sanitaria, campañas educativas y asistencia social de redes de contención. También, en la cuenta alta se usan los arroyos para la actividad ganadera; algunos productores manifiestan que dan de abrevar al ganado agua segura de perforaciones, aunque también están expuestas a los factores contaminantes.

A partir de esto, se identifica que la región aloja actividades incompatibles que compiten por el uso de los recursos suelo y agua. Se evidencia un territorio reconocido como frágil a partir de la ocupación de las planicies de inundación, con problemas de inundaciones cíclicas, generando un impacto negativo en los sectores residenciales, en las actividades industriales y el manejo y disposición de los residuos sólidos, y en la zona de ribera.

A su vez, el Gran La Plata tiene la complejidad de una región metropolitana, por lo que la interjurisdiccionalidad es determinante a la hora de planificar las acciones y gestionar el territorio, ya que las divisiones administrativas no coinciden con las unidades naturales, por lo que se dificulta la visualización y comprensión del problema. 
FIGURA 3

Síntesis de conflictos ambientales de las cuencas del Partido de La Plata

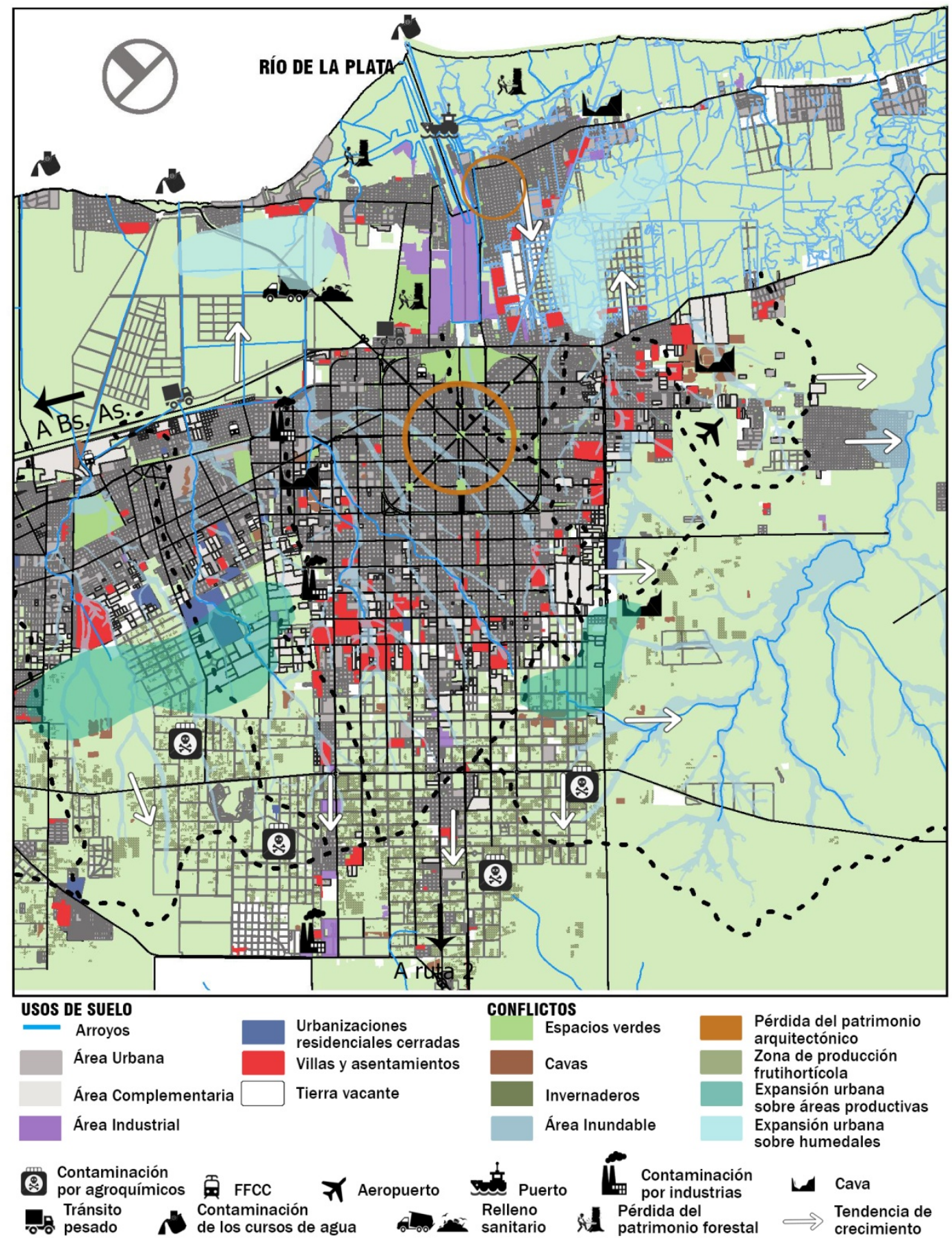

Elaboración propia, 2019

\section{Resultados}

A partir del reconocimiento de los conflictos en la región, se desarrolló la matriz de análisis de vulnerabilidad (Tabla 1). Mediante esta matriz se pudo identificar que la cuenca del Arroyo El Gato es la más vulnerable, 
incluyendo las subcuencas del arroyo El Gato, arroyo Pérez y el arroyo Regimiento. En segundo término se encuentra la cuenca del arroyo Maldonado (Figura 4).

En dichos casos los factores de susceptibilidad son los que tienen mayor impacto en la matriz de análisis, junto con la alteración del cauce y la impermeabilización de superficies. Estos factores deberían ser los focos de trabajo para reducir los niveles de vulnerabilidad de las cuencas más críticas. Asimismo, se encuentra como factor común a todas las cuencas una debilidad en los factores de resiliencia relacionados con infraestructura para prevenir inundaciones y con la gestión del riesgo, el acceso a información y los planes de contingencia.

Asimismo, se identificó la cuenca del Arroyo El Pescado como la cuenca con menor grado de vulnerabilidad, ya que se encuentra por fuera de la zona urbanizada y afecta a muy poca población y muy pocas actividades. Las demás cuencas presentan una vulnerabilidad media, por lo cual son cuencas en las que hay que poner atención, ya que si no se realizan acciones pueden aumentar el grado de vulnerabilidad, por el aumento de la exposición o de la susceptibilidad de la población.

TABLA 2

Factores causales de susceptibilidad del sistema de cuencas.

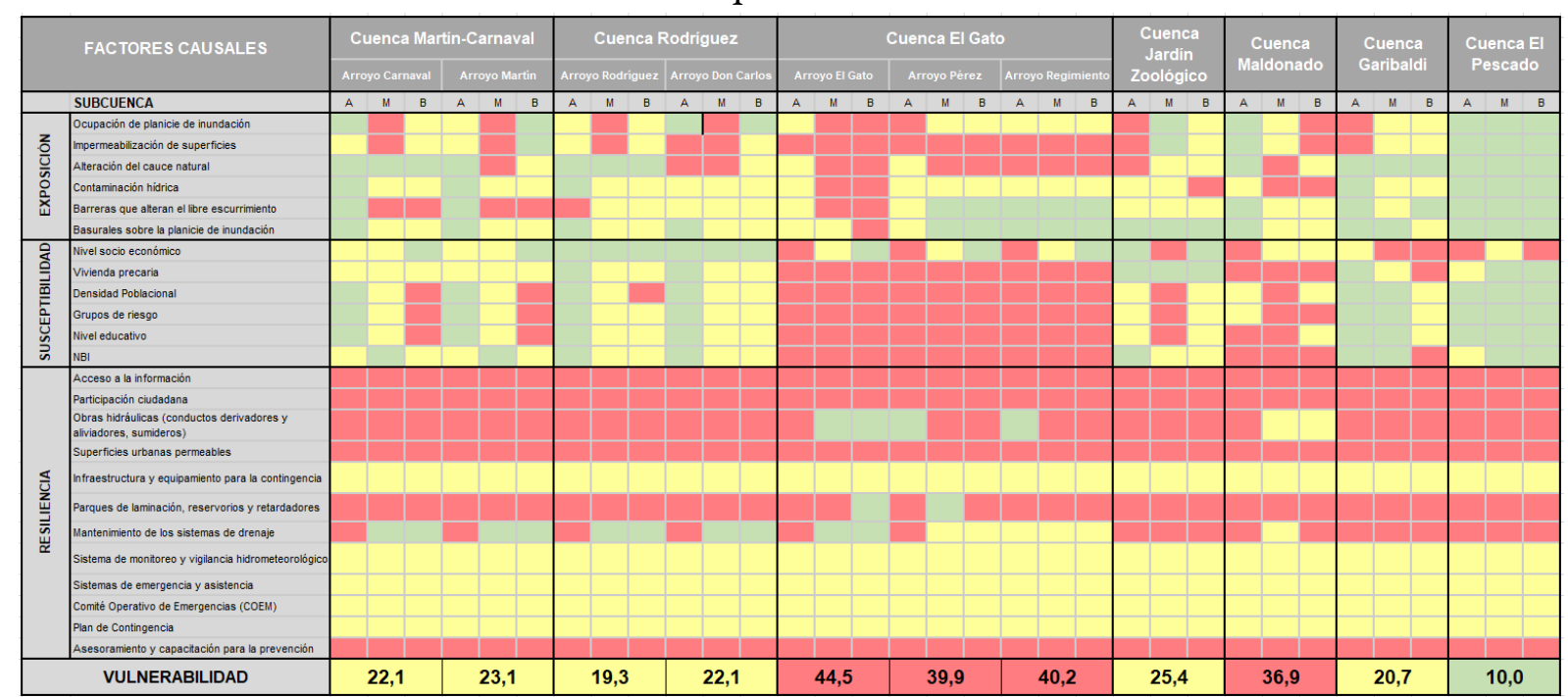

Elaboración propia, 2019 
FIGURA 4

Vulnerabilidad en las cuencas de la RGLP

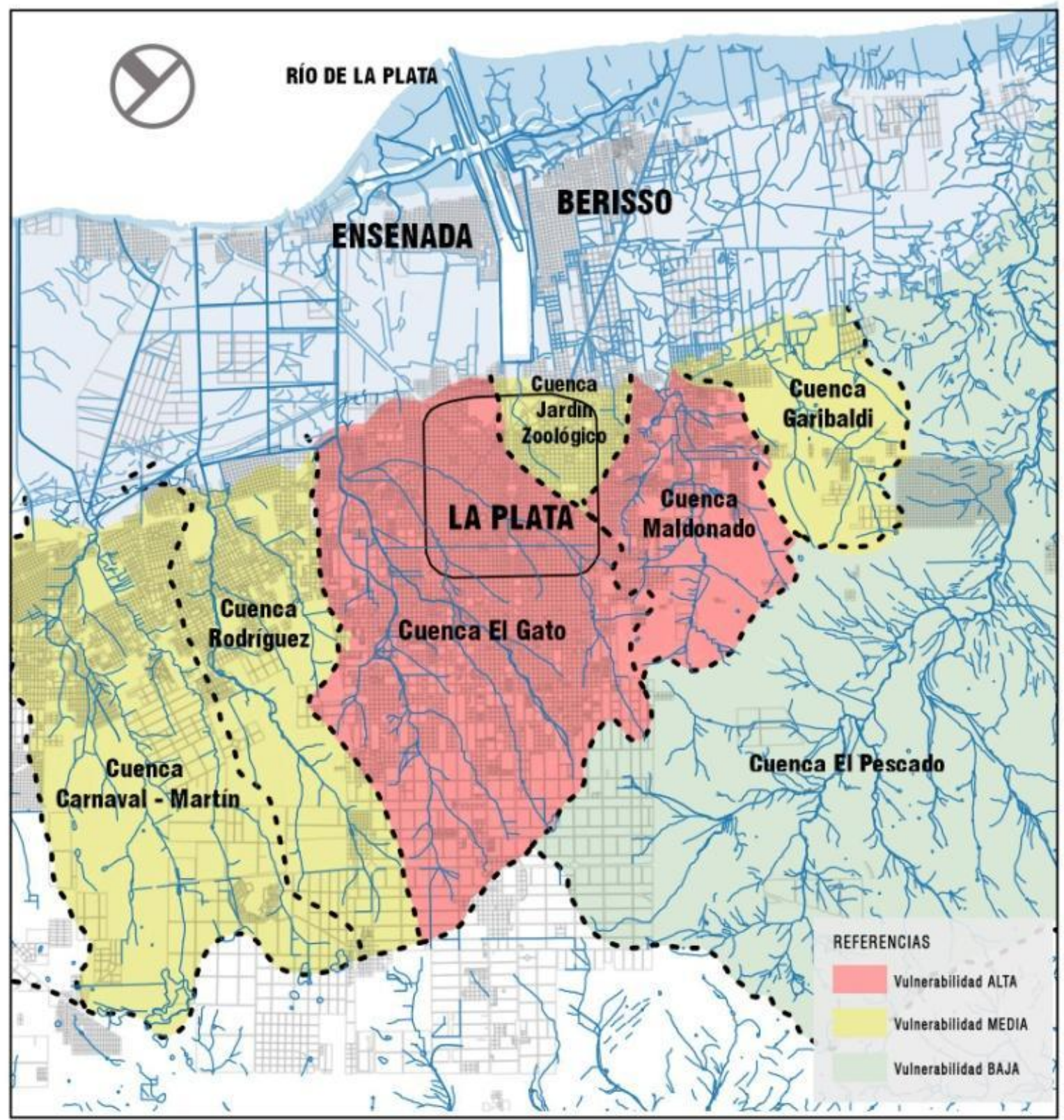

Elaboración propia, 2019

\section{Conclusiones}

La relación "agua-ciudad" en la RGLP siempre ha sido un punto conflictivo, incluso desde la fundación de la ciudad de La Plata. Ello, debido a que en la ocupación de la región, tanto en el casco fundacional como en las expansiones no planificadas del tejido de la periferia, no se tuvo en cuenta el soporte natural del territorio, lo que derivó en problemas urbano-ambientales en la actualidad.

Hablar de una visión integral que permita ver más allá de lo que se ve es mirar las interrelaciones que se dan entre los sistemas y entender el problema desde la reflexión, para generar una aproximación al lugar que brinde la premisa de cómo actuar sobre él para empezar a formar parte de un ecosistema en constante 
transformación. El desafío más importante es el de planificar coordinadamente tiempos diferenciados, como lo son el tiempo humano y el tiempo natural, pensando siempre en la idea de totalidad.

En este sentido, intervenir sobre el territorio desde la concepción de construcción social del riesgo implica reconocer la estrecha relación entre cultura y naturaleza, en la que lo más importante es construir nuevos vínculos entre el hombre y el medio que lo rodea. Y pensar las intervenciones futuras buscando una relación más armónica con el ambiente natural para reducir la vulnerabilidad frente a los fenómenos críticos que afectan la región.

\section{ReFERENCIAS}

Ábalos, I. (2005). Atlas Pintoresco Vol. 1: El observatorio. Barcelona: Gustavo Gili.

Consejo de Europa (2000). Convenio europeo del paisaje. Florencia: ISMA.

Herzer, H. (2011). Construcción del riesgo, desastre y gestión ambiental urbana: perspectivas en debate. Redesma, 5(2), 15-26.

Herzer, H. y Di Virgilio, M. (1996). Buenos Aires: pobreza e inundación. Eure, 22(67), 65-80.

Nogué, J. (2007). La construcción social del paisaje. Barcelona: Biblioteca Nueva.

Lavell, A. (2004). Vulnerabilidad social: Una contribución a la especificación de la noción y sobre las necesidades de investigación en pro de la reducción del riesgo. Disponible en INDECI: http://bvpad.indeci.gob.pe/doc/pdf/esp /doc852/doc852-contenido.pdf

Lynch, K. (1966). La imagen de la ciudad. Buenos Aires: Infinito.

Márquez, F. (2011). Planificación diseño y gestión participativa del paisaje. Buenos Aires: Nobuko.

Ravella, O. (2008). La ciudad entendida como un sistema complejo: estructura y tejido urbano. La Plata: Nobuko.

Roger, A. (2007). Breve tratado del paisaje. Madrid: Editorial Nueva.

Rogers, R. (2014). Ciudades para un pequeño planeta. Barcelona: Gustavo Gili.

Varela, L. (2001). Paisaje, reflexiones. La Plata: Al Margen. Colección Universitaria, Arquitectura.

\section{Notas}

1 Oficina para la reedición de riesgos de desastre de las Naciones Unidas - UNISDR. Terminología sobre Reducción de Riesgo de Desastres 2009 para los conceptos de amenaza, vulnerabilidad y riesgo.

\section{BY-NC-SA}

